\title{
Nutritional Biomarkers, Gene-Diet Interaction, and Risk Factors for Type 2 Diabetes
}

\author{
Ju-Sheng Zheng, ${ }^{1}$ Kaijun Niu, ${ }^{2}$ Simone Jacobs, ${ }^{3}$ Hassan Dashti, ${ }^{4}$ and Tao Huang ${ }^{5}$ \\ ${ }^{1}$ MRC Epidemiology Unit, University of Cambridge, Cambridge, UK \\ ${ }^{2}$ Nutritional Epidemiology Institute, Tianjin Medical University, Tianjin, China \\ ${ }^{3}$ Institute of Public Health, University of Heidelberg, Heidelberg, Germany \\ ${ }^{4}$ Center for Human Genetic Research, Massachusetts General Hospital, Harvard Medical School, Boston, MA, USA \\ ${ }^{5}$ Saw Swee Hock School of Public Health, National University of Singapore, Singapore
}

Correspondence should be addressed to Ju-Sheng Zheng; jusheng.zheng@mrc-epid.cam.ac.uk

Received 13 December 2016; Accepted 13 December 2016

Copyright (C) $2016 \mathrm{Ju}$-Sheng Zheng et al. This is an open access article distributed under the Creative Commons Attribution License, which permits unrestricted use, distribution, and reproduction in any medium, provided the original work is properly cited.

Prevalence of type 2 diabetes (T2D) has been increasing globally, gaining high public health priority [1]. T2D is a complex disease, resulting from an interplay between lifestyle and genetic factors. Evidence from several randomized controlled trials suggests that physical activity and dietary intervention could prevent or delay progression of T2D [2-4]. In addition, genome-wide association studies have identified hundreds of genetic variants associated with T2D [5]. In the present special issue, each paper investigated different aspects of T2D prevention strategy, ranging from physical activity and diet/nutritional biomarker to gene-epigenetic mechanisms, in animal models and human studies.

J. Qi et al. revealed that, in rats with insulin resistance induced by a high-fat diet, swimming exercise could improve insulin sensitivity, potentially via a reduction of TRIM72 expression, and upregulation of PI3-K/AKT signaling pathway, including IRS-1, p-AKT ${ }^{\text {Ser473 }}$, and AKT expression. The PI3-K/AKT signaling pathway is critically important for the regulation of insulin sensitivity and other biological processes related to aging $[6,7]$. This study further showcased the intricate mechanism linking physical activity (swimming in this study) to insulin resistance. With similar design in a highfat induced nonalcoholic fatty liver disease rodent model, Y. Xie et al. found that total alkaloid from Nelumbinis Plumula (NPA) could reduce insulin resistance via mechanism of restoring IRS1 and suppressing the JNK phosphorylation. Indeed, natural products derived from food resources have been proved to be promising natural remedies for $\mathrm{T} 2 \mathrm{D}$ prevention and treatment [8].

Objectively measured nutritional biomarker may overcome recall bias and measurement error resulting from traditional dietary assessment tools, such as food frequency questionnaires. Emerging metabolomics studies are revealing more and more promising nutritional biomarkers and linking them to health outcomes. One of the examples is BranchedChain Amino Acid (BCAA). In this issue, X. Zhao et al. systematically reviewed the current evidence on the relationship between BCAA and insulin resistance and concluded that circulating BCAAs (including valine, leucine, and isoleucine) are positively associated with insulin resistance and are useful biomarkers to predict future T2D cases. In a recent Mendelian randomization analysis, researchers identified a causal association of high circulating BCAAs with T2D risk and proved the causal role of BCAA metabolism in the T2D etiology [9]. Thus, use of nutritional biomarkers has obtained increasing popularity given their nature of objective measurement and potential usage to test causality in observational studies.

Lastly, L. Yang et al. updated the association of genetic variations in LEPR gene with T2D risk with a meta-analysis, providing robust evidence for the association between rs1137101 at LEPR and T2D risk. In addition, T. Matsha et al. demonstrated the role of DNA methylation-genetic variations in screen-detected diabetes or known diabetes on treatment in an African population. All the above efforts add 
to the current knowledge on the role of genetic and epigenetic modulation on T2D etiology [10].

Taken together, articles in this special issue highlight several important fields in the prevention and treatment of T2D, focusing on the important role of diet and physical activity, incorporating natural remedies from food resources and new omics (metabolomics and genomics) strategies. The issue also suggests the importance of integrative strategies for the prevention and treatment of T2D in future research.

Ju-Sheng Zheng
Kaijun Niu
Simone Jacobs
Hassan Dashti
Tao Huang

\section{References}

[1] NCD Risk Factor Collaboration (NCD-RisC), "Worldwide trends in diabetes since 1980: a pooled analysis of 751 population-based studies with 4.4 million participants," The Lancet, vol. 387, no. 10027, pp. 1513-1530, 2016.

[2] G. Li, P. Zhang, J. Wang et al., "Cardiovascular mortality, allcause mortality, and diabetes incidence after lifestyle intervention for people with impaired glucose tolerance in the $\mathrm{Da}$ Qing diabetes prevention study: a 23-year follow-up study," The Lancet Diabetes and Endocrinology, vol. 2, no. 6, pp. 474-480, 2014.

[3] E. S. Schellenberg, D. M. Dryden, B. Vandermeer, C. Ha, and C. Korownyk, "Lifestyle interventions for patients with and at risk for type 2 diabetes: a systematic review and meta-analysis," Annals of Internal Medicine, vol. 159, no. 8, pp. 543-551, 2013.

[4] A. Ramachandran, C. Snehalatha, S. Mary, B. Mukesh, A. D. Bhaskar, and V. Vijay, "The Indian Diabetes Prevention Programme shows that lifestyle modification and metformin prevent type 2 diabetes in Asian Indian subjects with impaired glucose tolerance (IDPP-1)," Diabetologia, vol. 49, no. 2, pp. 289297, 2006.

[5] D. Welter, J. MacArthur, J. Morales et al., "The NHGRI GWAS Catalog, a curated resource of SNP-trait associations," Nucleic Acids Research, vol. 42, no. 1, pp. D1001-D1006, 2014.

[6] S. Fröjdö, H. Vidal, and L. Pirola, "Alterations of insulin signaling in type 2 diabetes: a review of the current evidence from humans," Biochimica et Biophysica Acta (BBA)-Molecular Basis of Disease, vol. 1792, no. 2, pp. 83-92, 2009.

[7] E. M. Mercken, S. D. Crosby, D. W. Lamming et al., "Calorie restriction in humans inhibits the PI3K/AKT pathway and induces a younger transcription profile," Aging Cell, vol. 12, no. 4, pp. 645-651, 2013.

[8] J. L. Ríos, F. Francini, and G. R. Schinella, "Natural products for the treatment of type 2 diabetes mellitus," Planta Medica, vol. 81, no. 12-13, pp. 975-994, 2015.

[9] L. A. Lotta, R. A. Scott, S. J. Sharp et al., "Genetic predisposition to an impaired metabolism of the branched-chain amino acids and risk of type 2 diabetes: a Mendelian randomisation analysis," PLoS Medicine, vol. 13, no. 11, Article ID e1002179, 2016.

[10] E. Burgio, A. Lopomo, and L. Migliore, "Obesity and diabetes: from genetics to epigenetics," Molecular biology reports, vol. 42, no. 4, pp. 799-818, 2015. 


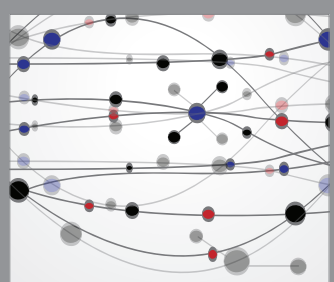

The Scientific World Journal
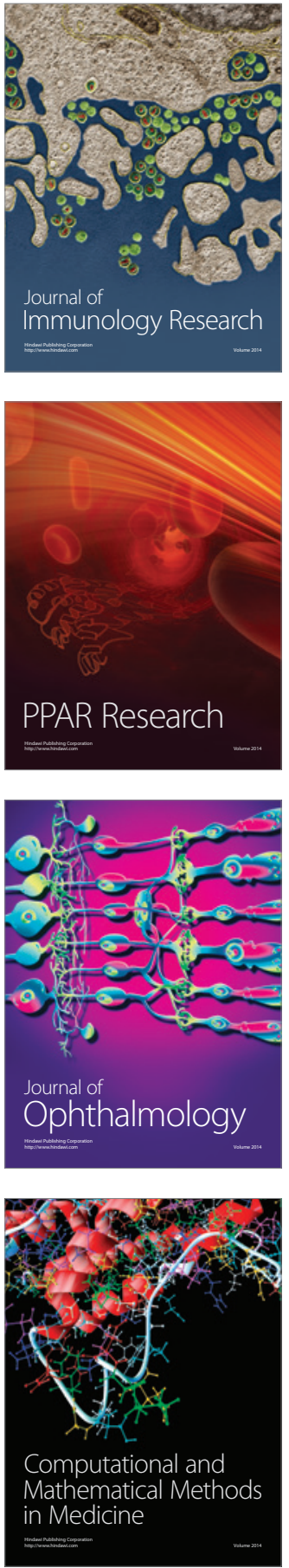

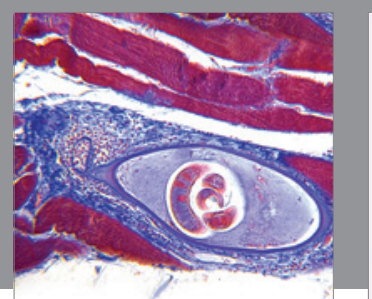

Gastroenterology Research and Practice

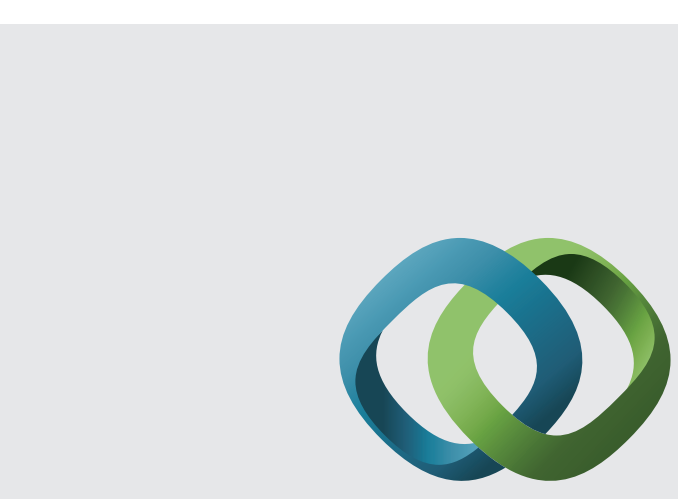

\section{Hindawi}

Submit your manuscripts at

http://www.hindawi.com
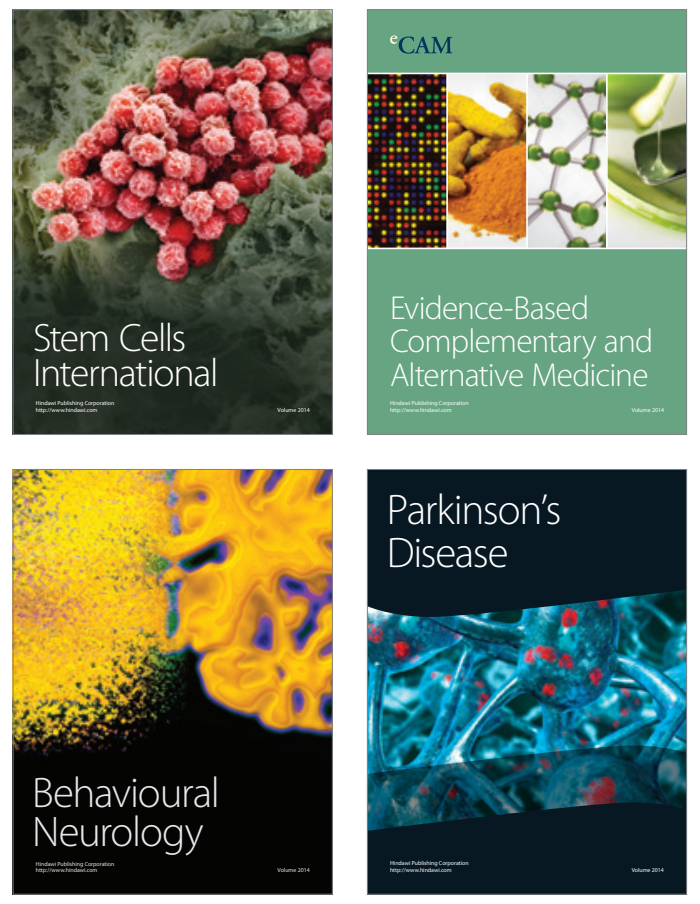
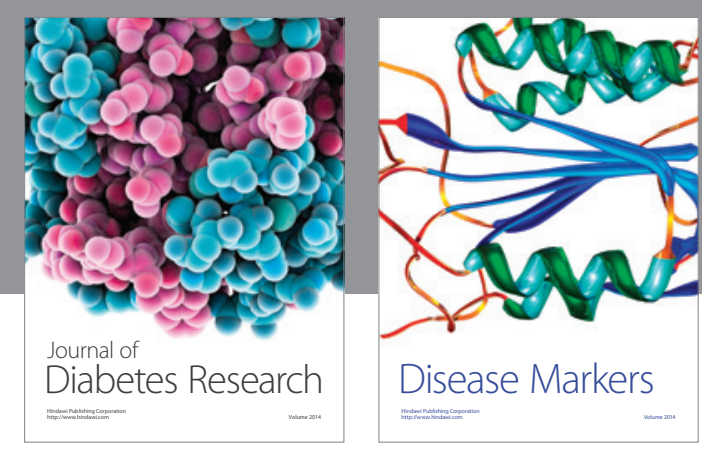

Disease Markers
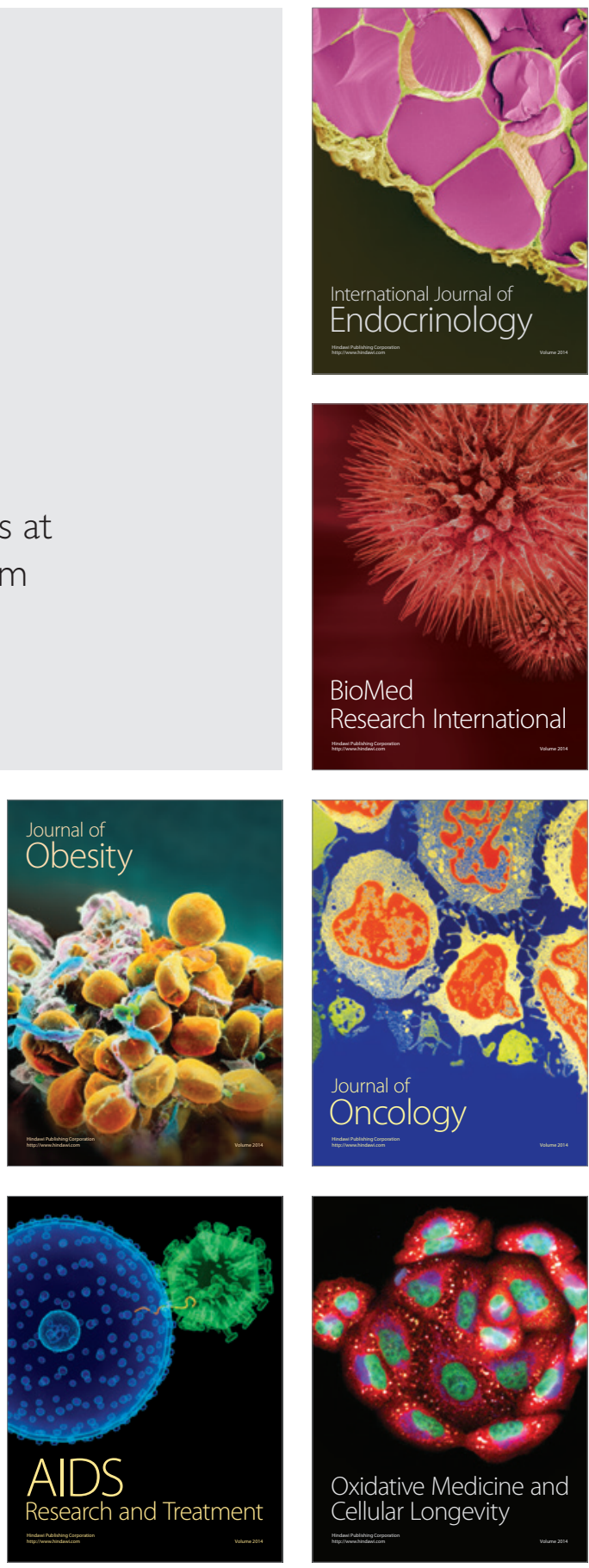